\title{
Light-responsive polymers for microfluidic applications
}

\author{
Jeroen ter Schiphorst, Janire Saez, Dermot Diamond, Fernando Benito-Lopez, Albertus P. H. J. Schenning
}

While the microfluidic device itself may be small, often the equipment required to control fluidics in the chip unit is large e.g. pumps, valves and mixing units, which can severely limit practical use and functional scalability. In addition, components associated with fluidic control of the device, more specifically the valves and pumps, contribute significantly to the overall unit cost. Here we sketch the problem of a gap be-tween high end accurate, but expensive sensor platforms, versus less accurate, but widely employable hand-held low-cost devices. Recent research has shown that the integration of light-responsive materials within microfluidic devices can provide the function of expensive fluidic components, and potentially en-able sophisticated measurements to be made using much less expensive equipment. An overview of the most recent developments will be presented for valves, mixers, transport and sample handling inside microfluidic devices.

\section{Introduction}

Microfluidic devices have been the subject of intense research activity the recent years, as they can allow complex measure-ments to be performed faster, with less waste generation, at lower cost, through miniaturisation and parallelisation of pro-cesses, resulting in highly efficient, high throughput and ac-curate and controllable analytical measurements. ${ }^{1-4}$ However, these devices typically require external components to fulfil critical functions, resulting in a chip-in-a-lab, rather than a lab-on-a-chip (Fig. 1).

This makes these devices limited for use in hand-held de-vices or in situ measurements, where interest is more towards large scale deployment with high sample frequency, rather than achieving the highest possible accuracy. A good example is the need for in situ monitoring of water quality in lakes or rivers, which at present is performed by manual sampling, typically once every 3 months, often missing spikes in pollu-tion or giving misleading information when sampling at a temporary point of high or low pollution. The ideal scenario is to deploy sensors at multiple locations to provide informa- tion for citizens through the internet. At present, this is limited by the high cost of the measuring platforms, which can be $>€ 20.000$ per unit. Clearly there is a need to drive down unit costs significantly, towards a more acceptable $€ 2000$ or less. In order to achieve this, new materials and concepts for microfluidic devices are required. ${ }^{5}$

Often, while the microfluidic device itself may be small, the equipment required to use the device itself is large, e.g. pumps, valves and mixing components. Moreover, the re-quirement of microscopes or other sophisticated optical or electrical elements for detection are drawbacks for the practi-cal use of microfluidic technology outside the laboratory. Therefore, the combination of detection units with conven-tional valves, pumps and mixers components, limits the scal-ability and prospects for the generation of relevant products based on microfluidics. Another key factor to be considered for autonomous devices placed in remote locations is the to-tal energy consumption of the device. That energy should be as low as possible in order to develop battery powered de-vices, capable of functioning indefinitely through localised energy scavenging.

Furthermore, an important factor to be considered, which is common for all the already cited requirements, is the limited use of new approaches to overcome these drawbacks, which unfortunately continue to appear in most of the micro-fluidic devices published in the literature. For instance, valves are often located off-chip, which is unscalable to more complex fluidic designs, and approaches that integrate large numbers of valves tend to require offchip connections for ac-tuation (e.g. pressure source). ${ }^{6}$ Combining fully integrating valves inside the channel with simple actuation control is 
Lab on a chip

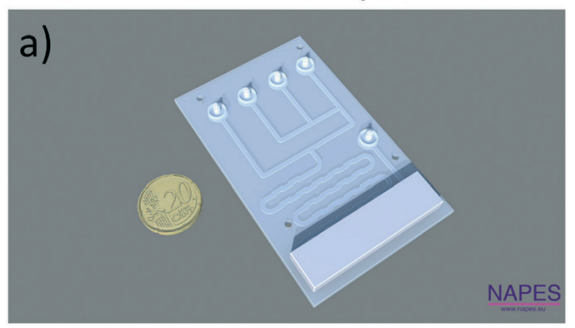

Chip with integrated valves

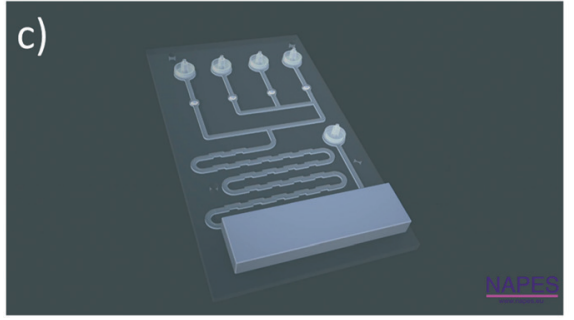

Chip in a lab

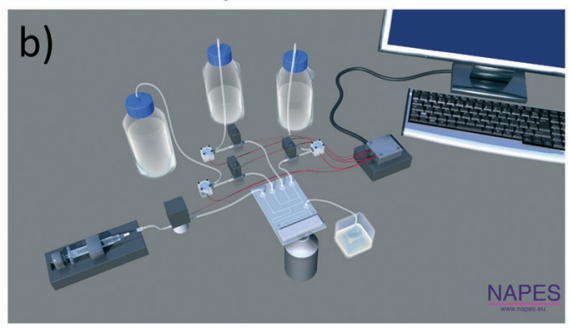

Integrated system

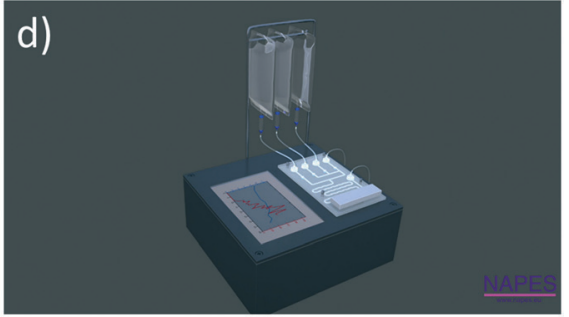

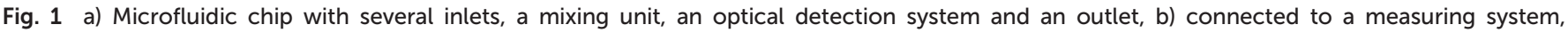

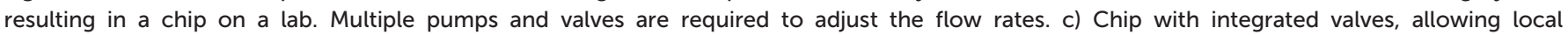

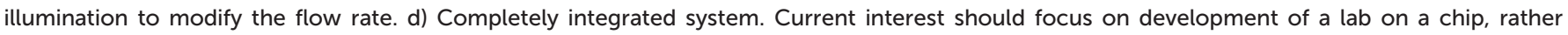
than a chip in a lab.

therefore a very attractive option for advancing microfluidics generally.

Recent research has shown that the integration of stimuliresponsive materials within microfluidic devices can lead to the development of novel in situ, microscale mechanical components with the potential to produce vastly simplified and highly efficient fluidic chip configurations. ${ }^{7-10}$ The use of integrated responsive materials requires less energy for operation and less dead volume through the elimination of significant amounts of tubing and inter-connects. The development of such smart devices reduces the unit cost, platform dimensions and complexity, in comparison to existing microfluidic devices. Such advances also open the way to a more modular approach to creating microfluidic devices, where the chip can be redesigned and replaced on the platform, allowing exchange of the chip unit and/or components in a cartridge form.

In analytical applications, microfluidic platforms typically perform several key operations. At the device front end, sampling, sample preparation, valving, mixing and transport is performed, while at the back end, analyte detection takes place. Stimuli-responsive materials can be used for several of these functions, and increasing interest in this topic is reflected in the rising number of publications in recent years, emerging since $2004 .{ }^{11}$ To actuate these materials, several stimuli have been used e.g. temperature, $\mathrm{pH}$, magnetic or electric and light. Light is particularly interesting, as it allows control over the components, without changing the interior conditions of the channel. Furthermore, light and temperature effects can be strongly linked, as light can also be used to induce a localised thermal response.

Several reviews on light-responsive materials and their in microfluidics have been published previously, ${ }^{8,12-17}$ and most recent overview of responsive materials for microfluidics was given by Hilber" ${ }^{18}$ in 2016. This review, entitled "Stimulusactive polymer actuators for next-generation microfluidic devices", highlighted the unsolved problems that still confront microfluidic technology. In that review the affirmation: "The operator may face difficulties in connecting the microfluidic device to ancillary hardware, such as external supplies, valves, pumps and other microfluidic devices", is highlighted, in accordance with our vision of generating microfluidic devices, where all functional parts reside inside the chip, rather than external. In this review, we will assess recent progress in light responsive materials for producing such internalised microfluidic components including microvalves and micromixers, along with the use of chemotactic microvehicles, responsive channel walls and sample handling to provide additional functionality.

\section{Microvalves}

Valves are used to ensure that liquids are at the correct place, at the correct time, within the microchannel network of a microfluidic device. Valves regulate fluid movement inside the microfluidic channel, determining whether or not a fluid is allowed to pass, as well as the amount of fluid that passes per unit time. Often, a microfluidic device is set-up in such a way, that fluids from different sources (e.g. sample, reagent) must be thoroughly mixed to enable subsequent detection by optical techniques. To achieve this, a microfluidic chip will require multiple valves and as the complexity of the system increases (e.g. multistage reagent addition, multicomponent analysis), valving requirements also become more demanding. Several valve configurations currently in common use are 
e.g. solenoid valves, screw valves, pneumatic valves ${ }^{6}$ and recently hydrogel valves. ${ }^{7,10}$ Depending on the applied pressures, desired accuracy and price that the user is willing to pay, the correct valve can be selected for the desired application. When multiple valves are needed in a microfluidic system, and low cost is important for the end-user, a low price for the valve is essential. In order to drive down the cost and energy consumption of the device, pneumatic and hydrogel valves are particularly interesting, as they can be applied cheaply and allow parallelisation without driving the price up too much. Hydrogel materials are based on the uptake and release of water, associated with the so-called lower critical solution temperature (LCST) behaviour. Below the LCST, the hydrogel absorbs water and swells, while above this temperature, the hydrogel releases the water and shrinks. A commonly used material is poly $N$-isopropylacrylamide (pNIPAM), a hydrogel with a LCST around $34^{\circ} \mathrm{C}$. Valves made of hydrogels are particularly interesting, as they frequently remain closed when not being addressed and can be opened with low energy light sources such as LEDs. Converting light to heat can also be employed to produce actuation in gels through LCST behaviour (Fig. 2a). ${ }^{19-21}$ Incorporating molecular photoswitches into the hydrogel produces similar actuation behaviour triggered by light (Fig. 2b). Although both approaches are viable and interesting, this review will focus particularly on photo-controlled gel actuation.

Recently, significant advances have been made to implement these valves in microfluidic devices for flow control, while Park and co-workers ${ }^{22}$ showed that these hydrogel materials can be polymerised in situ within microfluidic channels with high precision and controlled shape.

Sugiura and co-workers ${ }^{7}$ showed an elegant way of generating a photoresponsive microvalve that can be controlled by localised photopolymerisation (Fig. 2c). By incorporating a spiropyran based moiety in the hydrogel and flowing acidic water through the channel, the protonated merocyanine is produced, which is hydrophilic. This triggers switching of the chromophore to the predominantly hydrophilic from, with associated swelling of the hydrogel due to water uptake and closing of the microchannel. Under illumination with either blue or white light, the reverse behaviour occurred, with gel contraction and channel opening. Channel opening occurred within seconds, but closing required several hours, effectively limiting these valves to single use applications. Later, the same group developed an on-chip fluid control strategy for on-demand formation of arbitrary microchannels and parallel control of multiple microvalves using a computercontrolled micropatterned light irradiation on a photoresponsive hydrogel sheet. ${ }^{24}$

In addition to slow channel closing, an acidic environment was required, which is not compatible with many application scenarios, such as many biological processes that occur around neutral $\mathrm{pH}^{8}{ }^{8}$ Therefore, extending valve function of these materials to neutral $\mathrm{pH}$ is important. Ziółkowski and co-workers ${ }^{27}$ showed that the incorporation of acrylic acid in the backbone of the hydrogel allowed usage of these mate- a)

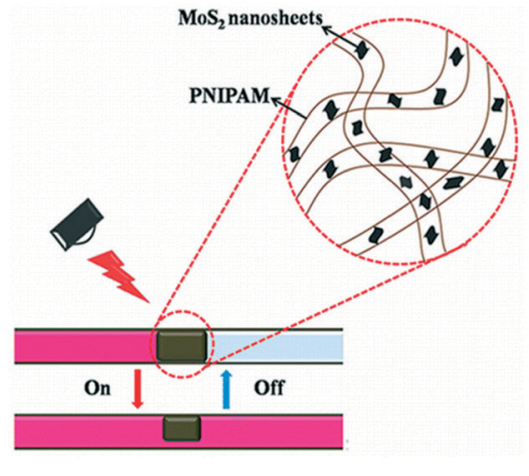

b)
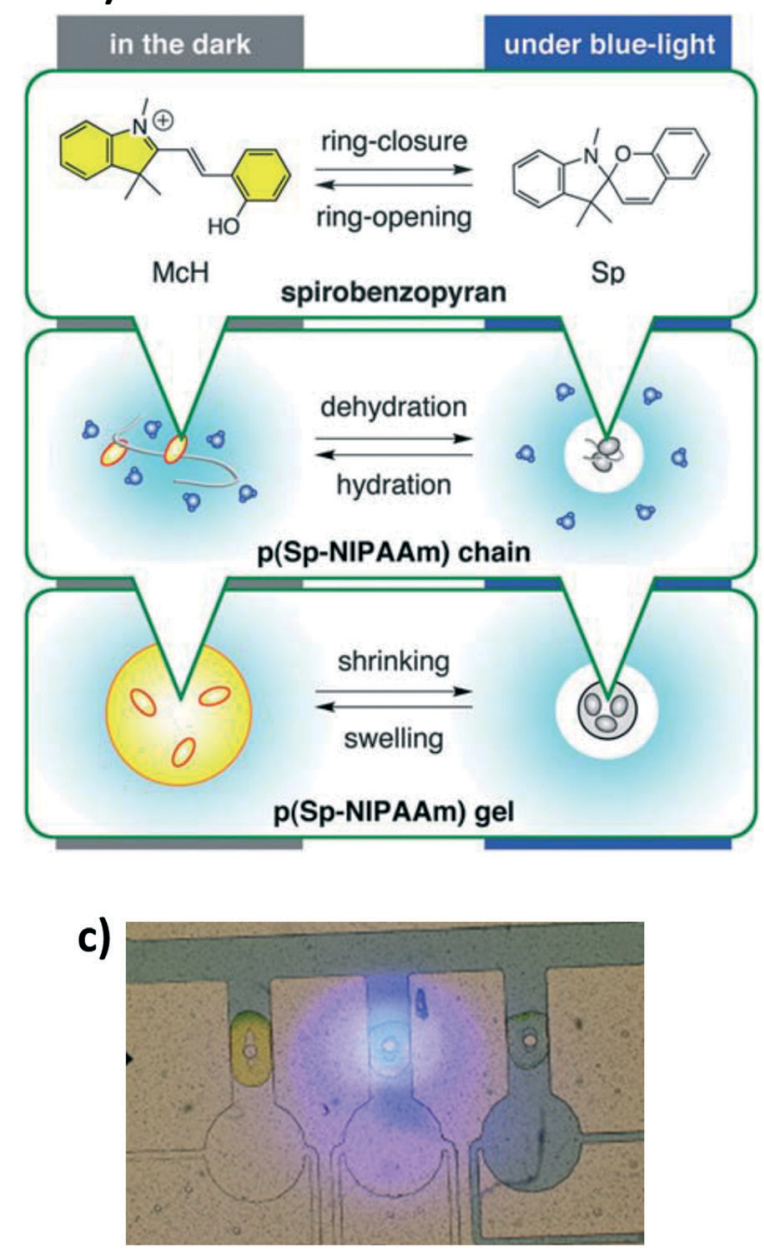

Fig. 2 a) Light responsive pNIPAM valve containing $\mathrm{MoS}_{2}$ nanosheets that can be heated by infrared light, resulting in photothermal opening of the valve. Adapted with permission from ref. 21. Copyright American Chemical Society 2016. b) Mechanism of light responsive hydrogel valves, where the isomerisation of spiropyran results in a polarity change. This change results in hydration or dehydration of a hydrogel chain. When copolymerised with a crosslinker, a gel that can shrink and swell by this principle is generated. Adapted with permission from ref. 23. Copyright 2011 Royal Society of Chemistry. Copyright 2017 American Chemical Society. c) Incorporation of light-responsive hydrogel valves in microchannels, showing the possibility to individually address these valves to let the fluid pass by (blue colour). Adapted with permission from ref. 7. Copyright 2007 Elsevier.

rials at neutral $\mathrm{pH}$, as the acid acts as an internal proton donor, providing the required protons to stabilise the 
protonated merocyanine. Furthermore, Satoh and coworkers $^{28}$ showed that the swelling of the hydrogel (and closing of the channel) could be sped up enormously by designing the chromophore correctly, whereby the isomerisation kinetics in both directions were within the same order of magnitude. This study together with the work of Ziolkowski and Sugiura where the foundation of the development of responsive hydrogels that allow manipulation of fluid within a timescale of seconds at high accuracy at neutral pH (Fig. 3a). Ter Schiphorst and co-workers ${ }^{10}$ identified that the rate determining step for gel shrinking was diffusion controlled, while gel swelling was limited by the molecular kinetics of the photoswitch isomerisation (Fig. 3b). They showed that combining above studies by designing spiropyrans derivatives with more rapid isomerisation kinetics produced gel valve structures that opened and closed within a few minutes, in great contrast of previous valves that required hours. This system employed a constant pressure head method for generating fluid movement in the microchannel. The speed of these valve can be even further optimised by down-scaling the gel valve in size, which reduces the mean diffusion path length, as well as producing molecular photoswitches with further enhanced isomerisation kinetics. As these hydrogel structures can be polymerised in situ within the channel, arrays of valves can be easily created that are individually addressed using appropriately positioned LEDs, allowing the array of valves that can be independently opened and closed. Coleman and coworkers $^{25}$ used a pulsating LED source to open and close the valve, or to regulate the flow rate by maintaining the valve at a state intermediate between fully open and closed, Fig. 3c. It was found that using various pulse sequences produced an oscillation in the flow rate of the microfluidic chip within seconds. The hydrogel tracked the pulse sequence of the LED and the final flow rate was found to be dependent on the on/off frequency and pulse duration of the light source. This allowed the flow rate to be controlled by varying the pulse frequency, whereby the hydrogel was operating out of equilibrium as it was not capable to equilibrate within the timeframe of the experiment. With recent developments in computer platforms, e.g. Raspberry Pi and Arduino systems, powerful tools to individually address, register and control the LED sources, as well as implementation of feedback loops, now allow the waveform of the incident light from LEDs to be precisely controlled, making it possible to address these valves individually and with high accuracy for a relatively low cost. Delaney and co-workers ${ }^{26}$ showed that these materials can be used for precision control of the flow rate in microfluidic channels. In this work, a modular platform was used, in which a photolayer containing multiple LEDs positioned adjacent to equivalent photoresponsive valve structures in a fluidic layer. A proportional integralderivative (PID) controller and a graphical user interface allowed the user to set the desired flow-pattern with very high precision, Fig. 3d. This system presented surprisingly high stability for the hydrogel and LED, showing that several a)

b)
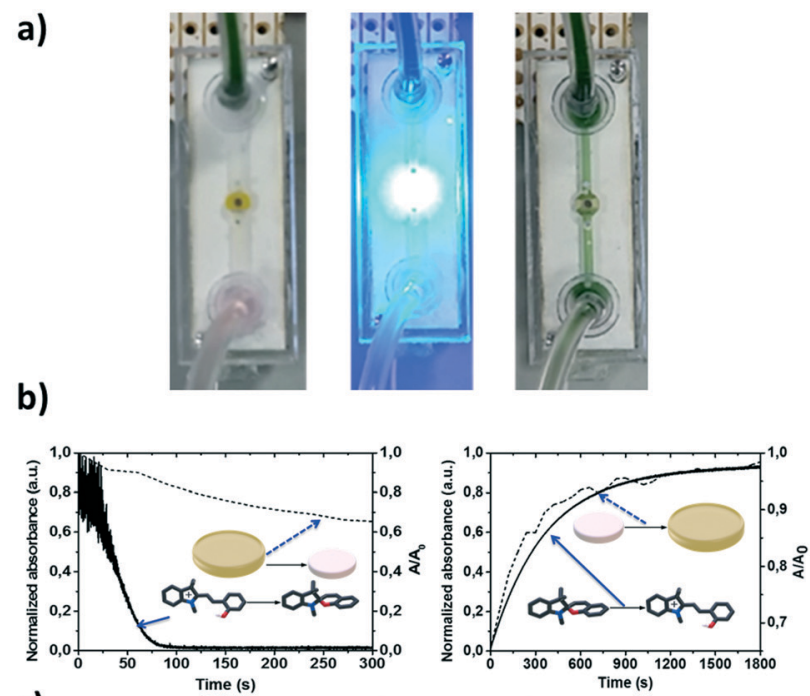

c)
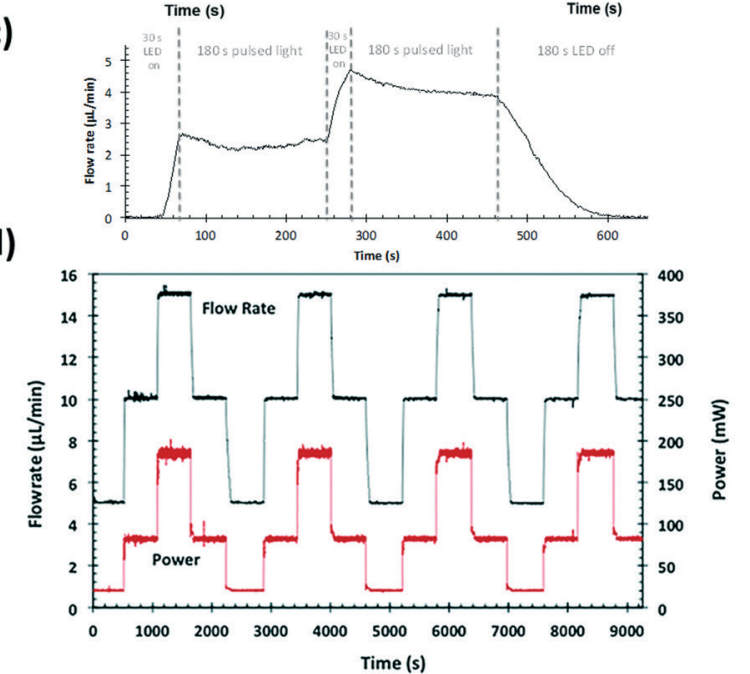

Fig. 3 The evolution of light responsive hydrogel valves. a) The valves developed by ter Schiphorst and co-workers, showing rapid opening upon illumination with blue light, but previously required several hours to close, working in an acidic environment. b) Comparison of molecular kinetics vs. macroscopic gel actuation, whereby the shrinking of the material is diffusion limited and the swelling isomerisation kinetics limited. $a$ and $b$ Adapted with permission from ref. 10. Copyright 2015 American Chemical Society. c) A valve based on a similar design as Sugiura, but containing a faster spiropyran moiety and working at neutral $\mathrm{pH}$. The effect of pulsating the light source on the flowrate, shows that relatively stable flows can be achieved using these systems. Adapted with permission from ref. 25. Copyright 2017 Elsevier. d) Fine-tuning of these valves using a feedback loop, allowing precise control over the flow rate. Adapted with permission from ref. 26. Copyright 2017 Royal Society of Chemistry.

hours of constant operation did not result in any drift of the flow rate or need to increase the source power.

As hydrogels tend to be rather soft materials, their ability to withstand higher pressures is limited. Various strategies can be employed to combat this limitation, one of which is to use ionogels rather than hydrogels. Ionogels are hybrid materials typically composed of a polymer and a room temperature ionic liquid (RTIL). When a RTIL is combined with a hydrogel, three-dimensional gel networks are generated, 
which acquire the physical and chemical properties of both components. ${ }^{29}$ Ionogels improve the mechanical and the viscoelastic properties of the hydrogel, which have important implications in the development of novel components for microfluidic devices. In order to synthesise light responsive ionogels, the ionogel can be photopolymerised with chromophoric moieties in a manner similar to the hydrogels described above. $^{30}$

Valves based on these photoresponsive ionogels presented fast actuation responses and response kinetics that could be varied by changing the RTIL in the ionogel. This chemical control of the ionogel and the actuation behaviour of the valves facilitates the generation of flexible, low-cost, and low power control units for liquid movement in microfluidic devices. This research was recently further expanded, showing that these valves can be used in a lab-on-a-disc configurations, combining the use of centrifugal forces to move the fluid from the centre of a disk to the outside of a disk with non-contact valve control which is compatible with the spinning disk. ${ }^{31}$

\section{Micromixers}

Valves and mixing in microfluidics often come hand in hand as many processes inside microfluidics involve joining multiple channels to combine fluids, and mixing these to a homogeneous state. In microchannels, laminar flow dominates and mixing occurs mostly by diffusion. For many assays, and particularly bioassays where large molecules are often involved, diffusion coefficients are low, with ineffective mixing resulting in poorly optimised processes and/or over-long assays. To overcome these problems, micromixers are used to more effectively mix laminar flows from merging channels. Efficient mixers based on active structures (i.e. structures that can be controlled) external to the microfluidic chip are well known in the literature. The ability to incorporate this function within the channels is obviously an attractive option. Simulations and experimental evidence show that asymmetric structures inside a channel result in efficient mixing of laminar streams. Stroock and co-workers ${ }^{32}$ showed that a staggered herringbone structure causes asymmetric circulation of the liquid and by alternating the asymmetric structure every cycle, an efficient micromixer was created that allowed fluids to be mixed within two centimetres, compared to a meter in the absence of this mixer. This work inspired many scientists to generate other micromixer designs for their specific set-up. However, as these structures are typically static, it does not allow the user to vary the structure configuration, unlike active micromixers. However, by incorporating responsive materials into channel walls, active mixers can be integrated into microfluidics systems, enabling the user to determine the degree of mixing. This was demonstrated by Prettyman and coworkers ${ }^{33}$ who showed that stimuli responsive hydrogels can be used to switch mixing on and off. They used $\mathrm{pH}$ responsive hydrogel posts arranged in a hexagonal oriented fashion. The diameter of the posts varied according to the fluid $\mathrm{pH}$, resulting in switching of mixing efficiency.

Van Schijndel and co-workers ${ }^{35}$ published a slanted groove mixer, a simplified version of the herringbone structured mixer, which further inspired ter Schiphorst and co-workers ${ }^{34}$ to use this design to generate a light-responsive micromixer. By filling the spaces between the slanted grooves with light responsive hydrogel, a structure was generated that mixed the fluids efficiently in the absence of light, as the inverse of the formed scaffold is formed by the swollen hydrogel. However, upon illumination with visible light, the hydrogel shrinks, resulting in an almost smooth channel surface and laminar flow (Fig. 4). The group of Baigl ${ }^{36}$ showed that it is possible to switch a stream of liquid from a tubing phase to a drop making phase (Fig. 5a). Shortly after, VenancioMarques and co-workers ${ }^{36}$ showed that mixing can also be achieved using the photoisomerisation of azobenzene (Fig. 5b). In this case, a flow focus device was created, wherein two oil phases (one containing a dye for visualisation) and an aqueous phase were brought in contact. The aqueous phase contained the light switchable chromophore, a water soluble trans-azobenzene. When not illuminated, the stable regime of the flow concentrator was tubing, in which the water phase was injected inside the oil phase. However, when illuminated with UV-light, the azobenzene isomerised to the cis form, resulting in a droplet regime. Due to the design of the channel, the droplets moved around chaotically, mixing the dyed and transparent streams. Continuing on this, Nurdin and co-workers ${ }^{37}$ showed that a similar system could be used to generate a high throughput switching system, capable of going from drop generation, to fusion and

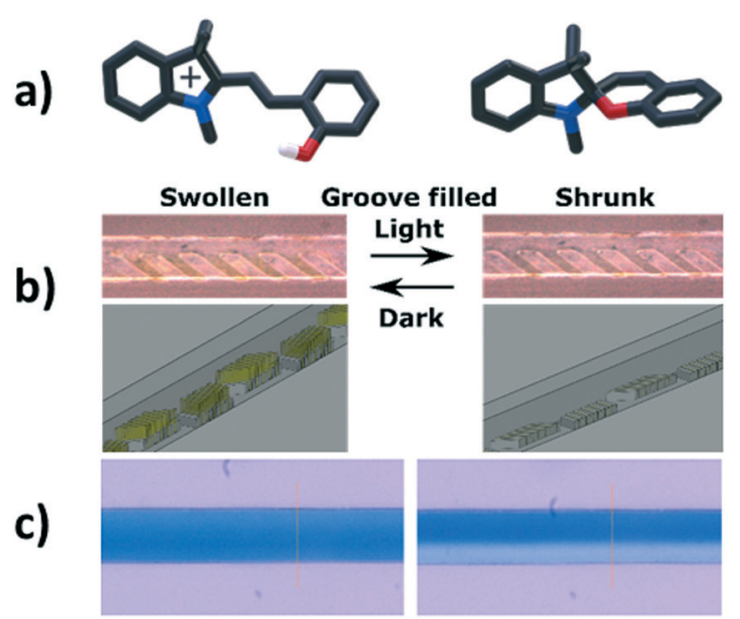

Fig. 4 Light responsive micromixer that can be switched. a) Isomerisation of the protonated merocyanine and spiropyran. b) Gel in the swollen and shrunk state, caused by illumination with white/blue light. c) Effect of illumination on the mixing behaviour of the micromixer. In the swollen state, the mixer is mixing the two water streams homogenously, while in the collapsed state, a clear separation between the fluids is visible. Adapted with permission from ref. 34 . Copyright 2017 John Wiley and Sons. 
a)
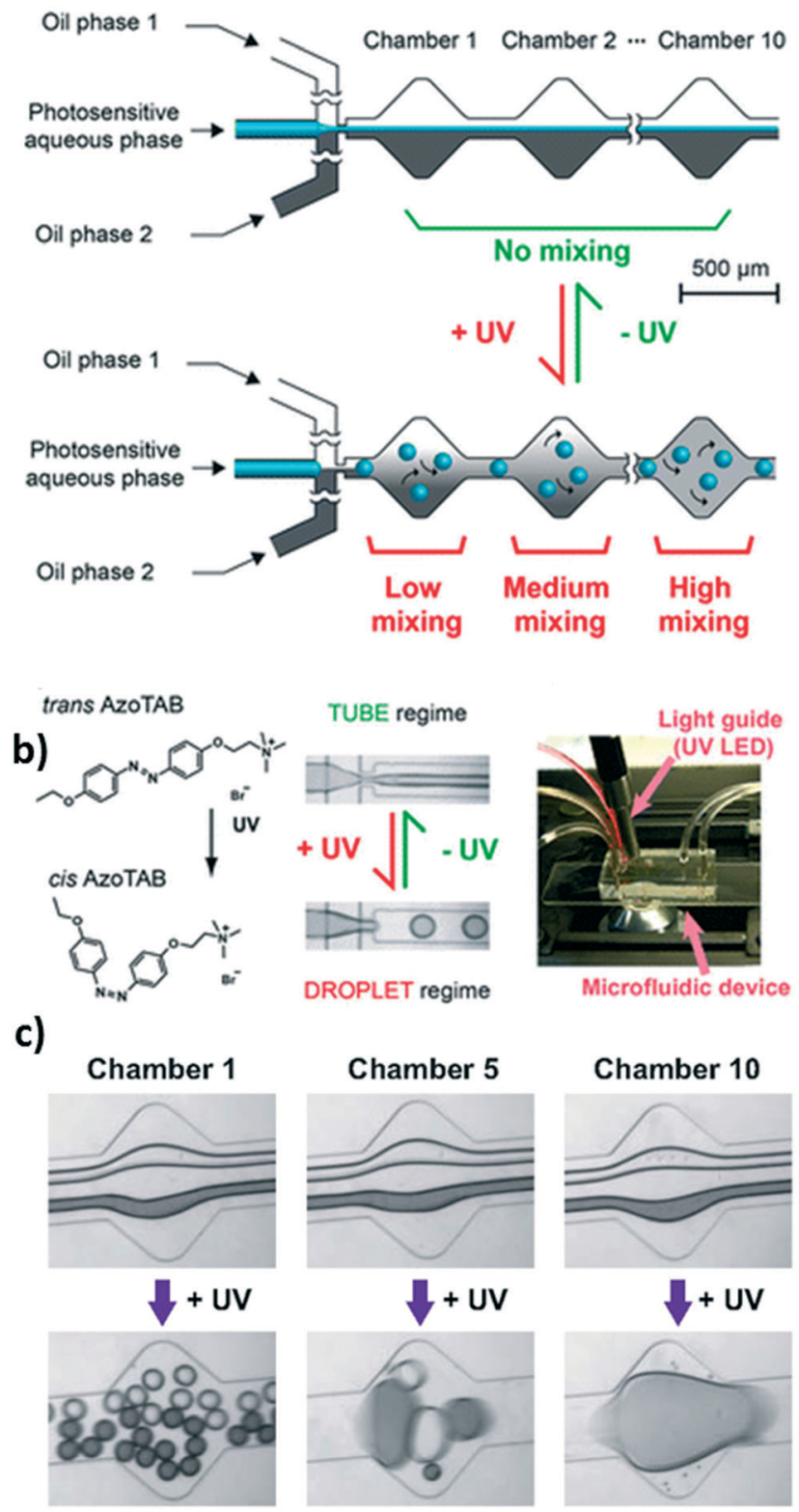

Fig. 5 a) Light responsive micromixer based on the capability to switch between a tubing and drop making system. This process is caused by the isomerisation of azobenzene b). When illuminated with UV-light, the azobenzene isomerises from trans to cis, causing a subtle change from jetting to drop making. Adapted with permission from ref. 36. Copyright 2013 American Chemical Society. c) Micromixer with two jets, showing the capability of parallelisation of this system. Adapted with permission from ref. 37. Copyright 2016 Elsevier.

mixing, using a dual flow focussing device, showing the versatility of this system (Fig. 5c).

\section{Fluid transport}

Transport of a fluid through a channel is a basic requirement in microfluidics in order to move samples and reagents from the front end to the back end of the device, where detection usually occurs. Between these parts of the microfluidic device, valving, pumping and mixing components are required to ensure sample transport. With the mind on widely deployable autonomous microfluidics, the use of several pumps, conventional electronic pumps, is limited and therefore, other ways of transporting liquids become mandatory, including moving micro vehicles in a closed fluid system, rather than moving the fluid through the channel. Hereby, chemotaxis and peristaltic motion are good examples to be integrated in microfluidic devices.

Chemotaxis is the movement of a substance to or away from a concentration gradient, based on the interaction of the substance to the gradient. This can be, for instance, a charged particle in a $\mathrm{pH}$ gradient. The use of chemotaxis comes from the necessity of moving liquids inside the microfluidic. Inside microfluidic devices, the laminar flow governs, so in order to generate movement of fluids/particles independently of the flow regime imposed by the microfluidic device configuration other type of approaches are needed. There are several ways of controlling fluid movements and transport with light, such as optically driven rotation of birefringent particles, ${ }^{38}$ optical alignment and spinning of laser-trapped microscopic particles, ${ }^{39}$ optical microrheology using rotating laser-trapped particles by using birefringent materials, ${ }^{40}$ optically driven pumps, ${ }^{41}$ chemopropulsion, ${ }^{42}$ and light driven electroosmotic flow, ${ }^{43}$ among others.

In particular, in chemopropulsion dependent systems, a fuel is required to create a driving force and move the microvehicle/container. This driving force can be provided by the generation of fluid shear forces through the motion of flagella $^{44,45}$ or propellers, ${ }^{46}$ by catalytic reactions, ${ }^{47}$ or bubble propulsion, ${ }^{48}$ and changes in surface tension. ${ }^{49,50}$ Despite its significant potential, there are relatively few examples of chemotaxis use in microfluidics due to the difficulty of controlling the chemistry of the process within the microfluidic environment. Nevertheless, there are interesting examples to be highlighted. For instance, the photodriven chemopropulsion described by Florea et al. $^{42}$ demonstrated that photo irradiation in close proximity to the micro-vehicle, in this case a lipophilic droplet of chromoionophore $\mathrm{I}(\mathrm{Cl})$ in 2-hexyldecanoic acid (HDA) initiated a rapid $\mathrm{pH}$ change in the aqueous solution due to conversion of protonated merocyanine to the deprotonated spiropyran isomer of water soluble $\mathrm{SP}_{-} \mathrm{SO}_{3} \mathrm{H}$. This localised photo-induced $\mathrm{pH}$ gradient causes a differential release of the deprotonated HDA (DA ${ }^{-}$is a very effective surfactant) at the droplet-aqueous boundary, and the droplets move along the channel apparently pushed by light (Fig. 6).

Despite the potential importance of light actuated micromotors, ${ }^{51}$ there are relatively few references to their use in microfluidic systems in the literature. Palagi et $a .^{52}$ developed photoresponsive soft micromotors actuated by light, where only the section of the motor irradiated with light deformed, whereas the non-irradiated section remained relaxed. The versatility of this actuation mechanism allows a single device to execute a variety of gaits including propulsive 


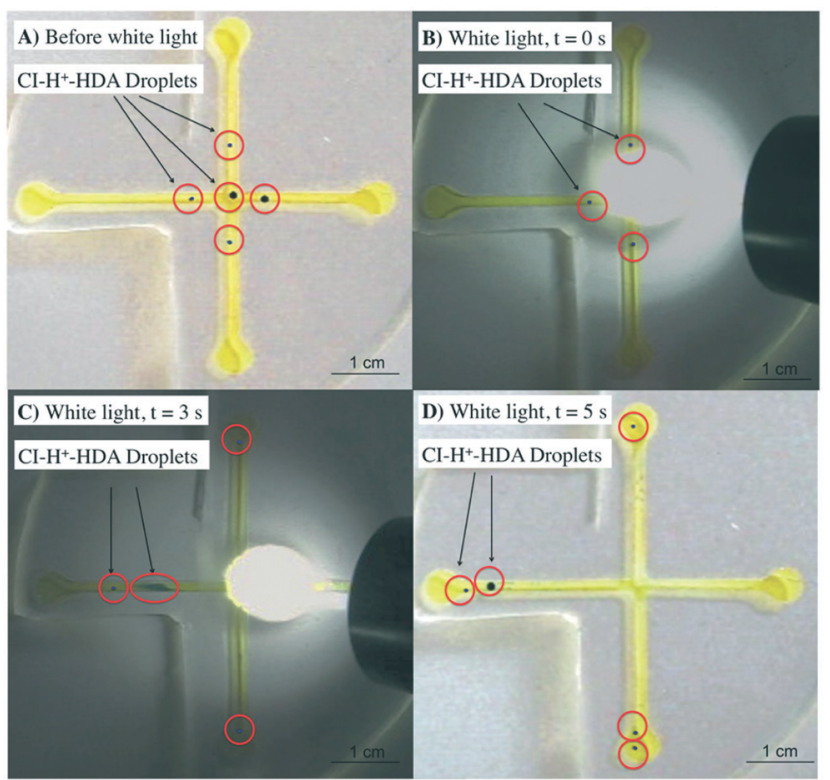

Fig. 6 Photograph of an open microfluidic channel with a cross shape to demonstrate the photo-chemopropulsion process. A) 5 droplets of different dimensions at the initial conditions. B) White light irradiation. C) After $3 \mathrm{~s}$ droplets move away from the light source. D) Droplets stop at the high $\mathrm{pH}$ region. There, the droplet boundary composition is stable. Adapted with permission from ref. 42. Copyright 2014 John Wiley and Sons.

motions that mimic the simplistic and antiplectic metachrony of ciliate protozoa.

Ibele et al. ${ }^{53}$ described the movement of silver chloride $(\mathrm{AgCl})$ micromotors with UV light irradiation. Recently, a new strategy was implemented for self-propulsion of polymeric tubular rockets by near-infrared (NIR). The rockets were functionalised with gold nanoshells, resulting in an asymmetric local heat distribution along the rockets that moved the rockets at speeds of up to $160 \mu \mathrm{m} \mathrm{s}^{-1}$. Moreover, the rockets could be started/stopped on demand by turning the NIR laser on and off. ${ }^{54} \mathrm{~A}$ similar investigation was published by Xuan and co-workers the same year. ${ }^{55}$

Self-organized microscale motors have also been described $^{56}$ some of which can be controlled by light. ${ }^{57,58}$ These micromotors can be used for cargo transport in microfluidic devices, or to break the laminar flow regime by linking these micromotors to promote mixing and liquid turbulence.

A seconds approach for transport is electroosmotic flow (EOF). Scarmagnani et al..$^{59}$ produced an acrylated-based spiropyran modified monolith integrated into a microfluidic device that could mediate EOF liquid movement. Under acidic conditions, the spiropyran acrylate monolith surface (SP) is preferably in the protonated merocyanine $\left(\mathrm{MC}-\mathrm{H}^{+}\right)$ form, which can be readily converted to the SP form through exposure to white light. When an external electric field is applied in the presence of $10^{-3} \mathrm{M} \mathrm{HCl}$, an EOF in the range of $0.7-1.6 \mu \mathrm{L} \mathrm{min}^{-1}$ is generated. Under constant white light irradiation, a significant decrease of the EOF occurs. Removal of the white light restored the flow rate to its original value, see Fig. 7.

Later, Walsh et al. ${ }^{43}$ showed that the flow rate could be increased or decreased using light, without having to change the applied field. When UV light is applied, the charged protonated MC form predominates and the EOF decreases by $50 \%$.

In nature, peristaltic motion is one of the most important movements performed by living organisms. For instance, muscular cells contract and relax following a specific direction, to control body movements. The possibility of generating peristaltic motion in synthetic materials using light will be a significant advance in microfluidic devices since pumping, which generally is driven by external electrical pumps, could then be integrated within the microfluidic device during the fabrication process. Maeda and co-workers ${ }^{60}$ demonstrated a light actuated material using peristaltic motion. A main drawback of this technique was that the actuation was controlled by on-off switching via an external output. Lately, the same group ${ }^{61-63}$ reported a synthetic NIPAMruthenium-based polymer gel whose motion was reminiscent of a living organism. The motion was induced by the Belosov-Zhabotinsky (BZ) reaction, which consists of a redox reduction of ruthenium that generates spatiotemporal dynamic patterns, chemical waves, under stationary conditions. This gel undergoes a metabolic cyclic reaction, induced by light, and could be integrated within microfluidic devices as a component for matter transport. The same BZ concept was used by Shinohara et al. ${ }^{64,65}$ for the development of a poly(NIPAM-Ru complex) gel that undergoes a cyclic swelling/shrinking change. In this case, the motion was achieved by the illumination with visible light, producing bromide ions, which can act as inhibitors of the oscillatory chemical reaction of the ruthenium complex.

This movement has been recently implemented into a microfluidic device by Francis et al. ${ }^{66}$ via a bipedal hydrogel based on $\mathrm{p}$ (NIPAM-co-SP-co-AA) polymer that reversibly shrinks and swells in aquatic environments in the presence or absence of white light. The actuation of the bipedal gel produces a walking motion on a ratcheted surface by taking a series of steps in a particular direction, see Fig. 8 .
(1)

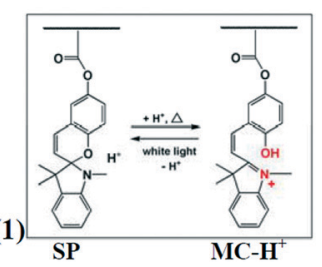

(2)

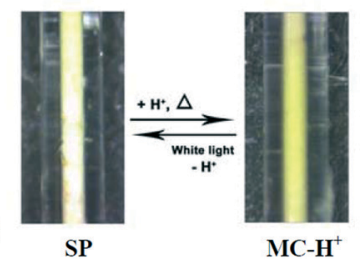

Fig. 7 (1) SP acrylate switching in acidic environment between the $\mathrm{MC}-\mathrm{H}^{+}$form (right) and the SP form (left) when irradiated with white light. (2) Picture of the spiropyran acrylate monolith flushed with $\mathrm{HCl}$ $10^{-3} \mathrm{M}$ switched between the $\mathrm{MC}-\mathrm{H}^{+}$form, in the absence of light exposure and the SP form when irradiated with white light. Adapted with permission from ref. 59. Copyright 2010 Trans Tech Publications. 

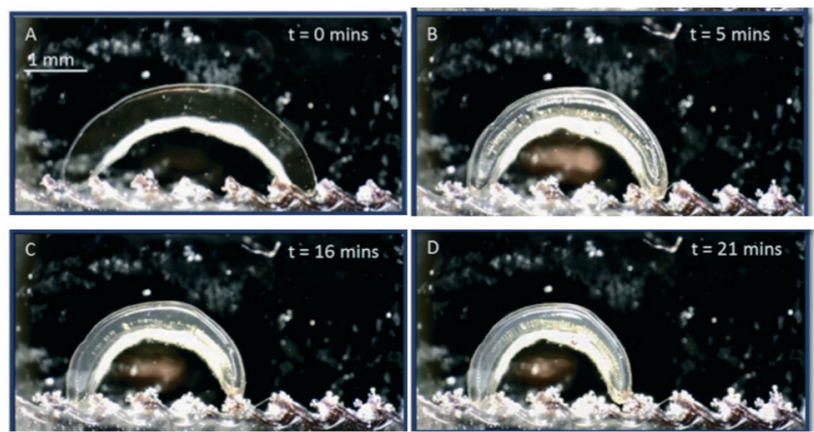

Fig. 8 Series of snapshots showing the behaviour of a hydrogel 'walker'. A and B shows contraction of the trailing leg. C-Swelling in the dark results in the forward leg being pushed over the surface. C and $D$ The sequence is repeated which results in the gel achieving a unidirectional walking motion (right to left). Adapted with permission from ref. 66. Copyright 2017 Elsevier.

This general approach can be extended to more advanced biomimetic walking (peristaltic) soft structures, which can perform a variety of tasks such as cargo transport and sensing in microfluidic devices.

\section{Photo-induced uptake, detection and release of metal ions}

The switchable mixing surfaces mentioned previously is an example of the increasing realisation that microfluidic channels have significant potential to perform important functions, beyond the current passive provision of a means for directing fluid movement. In particular, active sensing surfaces triggered by light are of great interest in microfluidics since this allows parts of the device to be activated/ deactivated with light.

For example, SP-based polymers can provide photoinduced uptake and release of guest ions on demand. The $\mathrm{MC}$ isomer can bind a range of different metal ions $\left(\mathrm{M}^{2+}\right)$, with the interaction taking place through the MC's phenolate oxygen. After MC complexation with metal ions, complexes can be converted to SP upon exposure to visible light, thereby resulting in the expulsion of the metal ions. This behaviour was nicely reviewed and explained by Klajn et al. ${ }^{17}$ An additional attractive feature is that the colour of the SP-coatings changes from colourless (predominantly SP) to purple (predominantly $\mathrm{MC}$ ) to a variety of other colours associated with the MC metal ion complexes. Therefore the coatings are inherently self-indicating of status, enabling simple confirmation of whether they are passive (SP), active (MC), or populated with bound ions. A variety of approaches have been reported in the literature including coatings;

- integrated onto silica beads, ${ }^{67,68}$ membranes or thin films. ${ }^{69}$

- integrated in the surface of the microchannels and fibers. $^{70,71}$ For instance, metals such as $\mathrm{Co}^{2+},{ }^{72-76} \mathrm{Zn}^{2+},{ }^{77,78}$ $\mathrm{Mg}^{2+},{ }^{29} \mathrm{Cu}^{2+},{ }^{79,80} \mathrm{Fe}^{2+}$ and $\mathrm{Ni}^{2+},{ }^{80}$ were detected (Fig. 9). The

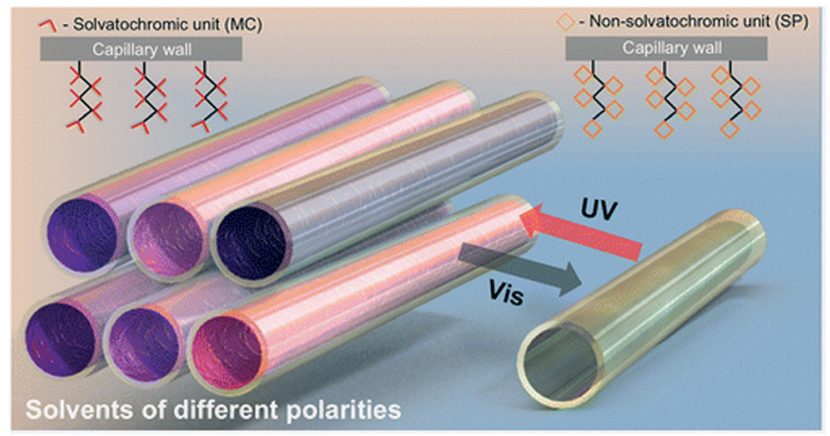

Fig. 9 Spiropyran functionalised capillary showing different responses based on different solvents, for optical detection. These capillaries can be embedded as a light responsive material, allowing on demand sensing. Adapted with permission from ref. 82. Copyright 2013 American Chemical Society.

same type of configuration was also use to detect and measure solvent polarities within a microfluidic device. ${ }^{81,82}$

Scarmagnani et $a .^{78}$ obtained spiropyran functionalised polystyrene microbeads, which can be reversibly switched between the colourless spiropyran to a highly purple coloured merocyanine by using UV light. These microbeads were successfully applied to the detection of $\mathrm{Cu}^{2+}$ ions by complexation with the merocyanine form. Later, the microbeads were integrated into a microcapillary using a monolithic polymer as a frit. A similar approach was followed by Byrne et al. ${ }^{73}$ complexing metal ions in poly(methyl methacrylate) (PMMA) slides. Both, microbeads and surfaces, can be regenerated several times allowing continuous ion uptake and release cycles. Following this work, Benito-Lopez et al. ${ }^{72}$ presented an array of microchannels in a PDMS microfluidic device, functionalised with a photochromic spiropyran. Upon exposure of light, the device displays photo-controlled uptake and release of metal ions such as $\mathrm{Co}^{2+}, \mathrm{Hg}^{2+}, \mathrm{Ca}^{2+}, \mathrm{Cu}^{2+}$ and $\mathrm{Zn}^{+2}$. Florea et al. ${ }^{75}$ made a step forward in the integration of sensing capabilities inside microcapillaries, functionalising them with a spiropyran polymer, using norbornyl derivative as monomer. In response of UV or white light, the spiropyran moiety was able to open and close within the polymer brushes. The micro-capillary systems were able to photo-bind and therefore detect divalent metals ions $\left(\mathrm{Zn}^{2+}, \mathrm{Co}^{2+}, \mathrm{Cu}^{2+}, \mathrm{Ni}^{2+}, \mathrm{Cd}^{2+}\right)$. The irradiation of the microcapillary with white light triggered the release of the metal ions and this difference can be measured by changes in the absorbance spectra. ${ }^{75,76}$

The same capillaries were also used as a tool for solvents polarity determination. ${ }^{77}$ Different solvents such as toluene, tetrahydrofuran, acetone, acetonitrile, ethanol and methanol were easily detected by irradiation with UV light. When in contact with the solvent, there is a change in colour of the microcapillary, which is unique for each polarity (Fig. 9).

\section{Conclusions and outlook}

Microfluidics is a booming technology that is establishing itself in many areas of research. However, there is a pressing 
need to integrate functions into microfluidics systems and significantly reduce unit prices while maintaining or enhancing reliability. If these advanced functions can be delivered with appropriate performance specifications, it will stimulate a much greater focus on creating microfluidic based instruments for in situ rather than in-lab measurements. Using light-responsive polymers, a large part of the expensive components can be replaced with lower cost materials that can be mass produced via photopolymerisation within the microfluidic system.

Such fully integrated photoswitchable valves and flow regulators represent a new scalable paradigm for microfluidics, enabling multiple components to be integrated into chips, in contrast to external conventional components, that must be connected using external tubing. Developing and commercialising a fabrication process to truly implement these valves in microfluidic systems would therefore be a logical future step. However, one should take into account that these valves, mostly based on hydrogels, also have several limitations. Firstly, these materials are soft, which limits the pressure it can withstand. Secondly, these materials are in direct contact with the medium, which can be problematic when the hydrogel absorbs components present in the fluid, that are subsequently released during contraction, leading to contamination and sample carry-over effects. Third, the hydrogels have only been investigated in water based media.

However, tremendous improvements have reported, going for instance from valves that operate over hours to valves that operate in minutes/seconds. We are therefore confident that continuing research will solve or reduce the impact of these limitations e.g. decoupling the valve from the analytical fluid by, for instance, placing a flexible protective membrane between the valve and the sample. This would allow the valve to swell and shrink for flow manipulation, while using and external placed reservoir for the hydration of the material, thus resolving the contamination issue. Similarly, tuning crosslinking or feature dimensions/shape should enable pressure resistance to be enhanced while simultaneously retaining fast actuation performance.

The challenge is to create microfluidic systems with much more sophisticated functionality than current approaches, but at an affordable or ideally dramatically reduced unit cost. We believe that this can be achieved with light responsive polymers and that they will play an important part in microfluidic devices. The ability to use a non-contact form of stimulation and control events in the fluidic system is very attractive as it is inherently non-invasive, and could simplify fabrication. Hereby the main field of interest is expected to form itself around low cost large scale applications, where inspiration should be drawn from biomimetic systems. Reliability will be key to generate these large scale applicability and idealistically a system that is self-aware of its conditions, and can autonomously self-maintain, repair and regulate, would appear to be needed.

Light responsive materials might find difficulties to compete to existing mechanical components in means of me- chanical stability, but will excel in their use for mass parallel systems. The possibility to combine computational platforms with cheap LED technology, and precise control over the gel size is exciting and a field of research that is currently underexposed. To develop the next generation of analytical devices, a molecule to device approach should be used. New molecules are designed, to fulfil the requirements of a material. These materials are integrated into a (microfluidic) device. Hereby the main topics are represented as materials, sensing, microfluidics and integration. These fields are currently experiencing a dramatic rate of continuous innovation as shown in this review and provide the highest potential of forthcoming breakthroughs for a technological revolution. Big steps towards truly developing these devices and in-field employment will only continue when there is close collaboration between scientists in various scientific disciplines and commercial entities to create the next generation of light responsive microfluidic devices. We hope this review will inspire scientists working in the fields of mechanical engineering, materials science, analytical chemistry and microfluidics to work together to deliver creative solutions to these issues, and thereby open greatly expanded possibilities for microfluidics in the future.

\section{Acknowledgements}

The authors would like to acknowledge the many discussions and contributions with all of our former and current colleagues. Their names are given in the references cited. A special word of thanks is expressed to all "NAPES" members for many inspiring discussions and fruitful collaborations. The authors acknowledge funding for this research under the European Union's Seventh Framework Programme (FP7) for research, technological development and demonstration, through the NAPES project grant agreement no. 604241. F. B.-L. acknowledges funding support from the Ramón y Cajal Programme (Ministerio de Economía y Competitividad), Spain as well as Gobierno Vasco, Dpto. Industria, Innovacion, Comercio y Turismo under ELKARTEK 2016 with Grant No. KK-2015/0000088 and Gobierno de España, Ministerio de Economía y Competitividad, with Grant No. BIO2016-80417P. We also acknowledge support from Science Foundation Ireland under the INSIGHT Centre initiative (SFI/12/RC/2289) and Enterprise Ireland (IP 2016 0502).

\section{Notes and references}

1 G. M. Whitesides, Nature, 2006, 442, 368-373.

2 C.-Y. Lee, C.-L. Chang, Y.-N. Wang and L.-M. Fu, Int. J. Mol. Sci., 2011, 12, 3263-3287.

3 E. A. Mansur, M. Ye, Y. Wang and Y. Dai, Chin. J. Chem. Eng., 2008, 16, 503-516. 
4 H. E. H. Meijer, M. K. Singh, T. G. Kang, J. M. J. Den Toonder and P. D. Anderson, Macromol. Symp., 2009, 279, 201-209.

5 D. Diamond, R. Byrne, F. B. Lopez, J. Cleary, D. Maher, J. Healy, J. Kim and K. Lau, IEEE SENSORS 2010 Conf., 2010, pp. 1079-1082.

6 M. A. Unger, H. P. Chou, T. Thorsen, A. Scherer and S. R. Quake, Science, 2000, 288, 113-116.

7 S. Sugiura, K. Sumaru, K. Ohi, K. Hiroki, T. Takagi and T. Kanamori, Sensors Actuators A Phys., 2007, 140, 176-184.

8 L. Florea, D. Diamond and F. Benito-Lopez, Macromol. Mater. Eng., 2012, 297, 1148-1159.

9 D. J. Beebe, J. S. Moore, J. M. Bauer, Q. Yu, R. H. Liu, C. Devadoss and B. Jo, Nature, 2000, 404, 588-590.

10 J. ter Schiphorst, S. Coleman, J. E. Stumpel, A. Ben Azouz, D. Diamond and A. P. H. J. Schenning, Chem. Mater., 2015, 27, 5925-5931.

11 (data gained from web of knowledge citation report stimuli responsive materials). 20 publications in 2004, 474 publications in 2017.

12 D. Eddington, Adv. Drug Delivery Rev., 2004, 56, 199-210.

13 C. Zhang, D. Xing and Y. Li, Biotechnol. Adv., 2007, 25, 483-514.

14 I. Tomatsu, K. Peng and A. Kros, Adv. Drug Delivery Rev., 2011, 63, 1257-1266.

15 D. Baigl, Lab Chip, 2012, 12, 3637-3653.

16 A. Döring, W. Birnbaum and D. Kuckling, Chem. Soc. Rev., 2013, 42, 7391-7420.

17 R. Klajn, Chem. Soc. Rev., 2014, 43, 148-184.

18 W. Hilber, Appl. Phys. A: Mater. Sci. Process., 2016, 122, 1-39.

19 A. D. Jadhav, B. Yan, R. Luo, L. Wei and X. Zhen, Biomicrofluidics, 2015, 9, 34114.

20 C. Zhu, Y. Lu, J. Peng, J. Chen and S. Yu, Adv. Funct. Mater., 2012, 22, 4017-4022.

21 J. Zhang, P. Du, D. Xu, Y. Li, W. Peng, G. Zhang, F. Zhang and X. Fan, Ind. Eng. Chem. Res., 2016, 55, 4526-4531.

22 S. Park, D. Kim, S. Y. Ko, J.-O. Park, S. Akella, B. Xu, Y. Zhang and S. Fraden, Lab Chip, 2014, 14, 1551-1563.

23 T. Satoh, K. Sumaru, T. Takagi and T. Kanamori, Soft Matter, 2011, 7, 8030-8034.

24 S. Sugiura, A. Szilágyi, K. Sumaru, K. Hattori, T. Takagi, G. Filipcsei, M. Zrínyi and T. Kanamori, Lab Chip, 2009, 9, 196-198.

25 S. Coleman, J. ter Schiphorst, A. Ben Azouz, S. Bakker, A. P. H. J. Schenning and D. Diamond, Sens. Actuators, B, 2017, 245, 81-86.

26 C. Delaney, P. Mccluskey, S. Coleman, J. Whyte, N. Kent and D. Diamond, Lab Chip, 2017, 17, 2013-2021.

27 B. Ziółkowski, L. Florea, J. Theobald, F. Benito-Lopez and D. Diamond, Soft Matter, 2013, 9, 8754-8760.

28 T. Satoh, K. Sumaru, T. Takagi, K. Takai and T. Kanamori, Phys. Chem. Chem. Phys., 2011, 13, 7322-7329.

29 M. Czugala, C. O'Connell, C. Blin, P. Fischer, K. J. Fraser, F. Benito-Lopez and D. Diamond, Sens. Actuators, B, 2014, 194, 105-113.

30 F. Benito-Lopez, R. Byrne, A. M. Răduță, N. E. Vrana, G. McGuinness and D. Diamond, Lab Chip, 2010, 10, 195-201.
31 J. Saez, T. Glennon, M. Czugala, A. Tudor, J. Ducreé, D. Diamond, L. Florea and F. Benito-lopez, Sens. Actuators, B, 2018, 257, 963-970.

32 A. D. Stroock, S. K. W. Dertinger, A. Ajdari, I. Mezic, H. A. Stone and G. M. Whitesides, Science, 2002, 295, 647-652.

33 J. B. Prettyman and D. T. Eddington, Sens. Actuators, B, 2011, 157, 722-726.

34 J. ter Schiphorst, G. G. Melpignano, H. E. Amirabadi, M. H. J. M. Houben, S. Bakker, J. M. J. den Toonder and A. P. H. J. Schenning, Macromol. Rapid Commun., 2018, 39, 1700086.

35 T. van Schijndel, M. K. Singh, M. Gillies, N. Kahya, A. Kharin and J. M. J. den Toonder, Macromol. Mater. Eng., 2011, 296, 373-379.

36 A. Venancio-Marques, F. Barbaud and D. Baigl, J. Am. Chem. Soc., 2013, 135, 3218-3223.

37 L. Nurdin, A. Venancio-Marques, S. Rudiuk, M. Morel and D. Baigl, C. R. Chim., 2015, 19, 199-206.

38 T. Wu, T. A. Nieminen, S. Mohanty, J. Miotke, R. L. Meyer, H. Rubinsztein-dunlop and M. W. Berns, Nat. Photonics, 2011, 6, 62-67.

39 M. E. J. Friese, T. A. Nieminen, N. R. Heckenberg and H. Rubinsztein-Dublop, Nature, 1998, 395, 348-350.

40 A. I. Bishop, T. A. Nieminen, N. R. Heckenberg and H. Rubinsztein-dunlop, Phys. Rev. Lett., 2004, 92, 14-17.

41 J. Leach, H. Mushfique and J. Cooper, Lab Chip, 2006, 6, 735-739.

42 L. Florea, K. Wagner, P. Wagner, G. G. Wallace, F. Benito-Lopez, D. L. Officer and D. Diamond, Adv. Mater., 2014, 26, 7339-7345.

43 Z. Walsh, S. Scarmagnani, F. Benito-lópez, S. Abele, F. Nie, C. Slater, R. Byrne, D. Diamond, B. Paull and M. Macka, Sens. Actuators, B, 2010, 148, 569-576.

44 S. Nain and N. N. Sharma, Front. Life Sci., 2015, 8, 2-17.

45 D. B. Weibel, P. Garstecki, D. Ryan, W. R. Diluzio, M. Mayer, J. E. Seto and G. M. Whitesides, Proc. Natl. Acad. Sci. U. S. A., 2005, 102, 11963-11967.

46 G. Vizsnyiczai, G. Frangipane, C. Maggi, F. Saglimbeni, S. Bianchi and R. Di Leonardo, Nat. Commun., 2017, 8, 1-7.

47 R. Dong, Y. Hu, Y. Wu, W. Gao, B. Ren, Q. Wang and Y. Cai, J. Am. Chem. Soc., 2017, 139, 1722-1725.

48 F. Mou, Y. Li, C. Chen, W. Li, Y. Yin, H. Ma and J. Guan, Small, 2015, 11, 2564-2570.

49 W. Li, X. Wu, H. Qin, Z. Zhao and H. Liu, Adv. Funct. Mater., 2016, 26, 3164-3171.

50 S. D. Janssens, S. Koizumi, E. Fried, S. D. Janssens, S. Koizumi and E. Fried, Phys. Fluids, 2017, 29, 32103.

51 G. K. Knopf and Y. Otani, Optical Nano and Micro Actuator Technology, CRC Press, 2012.

52 S. Palagi, A. G. Mark, S. Y. Reigh, K. Melde, T. Qiu, H. Zeng, C. Parmeggiani, D. Martella, A. Sanchez-castillo, N. Kapernaum, F. Giesselmann, D. S. Wiersma, E. Lauga and P. Fischer, Nat. Mater., 2016, 15, 647-654.

53 M. Ibele, T. E. Mallouk and A. Sen, Angew. Chem., Int. Ed., 2009, 48, 3308-3312.

54 Z. Wu, T. Si, W. Gao, X. Lin, J. Wang and Q. He, Small, 2016, 12, 577-582. 
55 M. Xuan, Z. Wu, J. Shao, L. Dai, T. Si and Q. He, J. Am. Chem. Soc., 2016, 138, 6492-6497.

56 W. Gao, A. Pei, X. Feng, C. Hennessy and J. Wang, J. Am. Chem. Soc., 2013, 135, 998-1001.

57 P. K. Kundu, S. Das, J. Ahrens and R. Klajn, Nanoscale, 2016, 8, 19280-19286.

58 L. Wang and S. Sánchez, Lab Chip, 2015, 15, 4383-4386.

59 S. Scarmagnani, Z. Walsh, F. Benito-Lopez, M. Macka and D. Diamond, Adv. Sci. Technol., 2010, 76, 100-105.

60 S. Maeda, Y. Hara, T. Sakai, R. Yoshida and S. Hashimoto, Adv. Mater., 2007, 19, 3480-3484.

61 S. Maeda, Y. Hara, R. Yoshida and S. Hashimoto, Angew. Chem., Int. Ed., 2008, 47, 6690-6693.

62 S. Maeda, Y. Hara, R. Yoshida and S. Hashimoto, Macromol. Rapid Commun., 2008, 29, 401-405.

63 S. Maeda, Y. Hara, R. Yoshida and S. Hashimoto, Int. J. Mol. Sci., 2010, 11, 52-66.

64 S. Shinohara, T. Seki, T. Sakai, R. Yoshida and Y. Takeoka, Angew. Chem., Int. Ed., 2008, 47, 9039-9043.

65 S. Shinohara, T. Seki, T. Sakai, R. Yoshida and Y. Takeoka, Chem. Commun, 2008, 0, 4735-4737.

66 W. Francis, A. Dunne, C. Delaney, L. Florea and D. Diamond, Sens. Actuators, B, 2017, 250, 608-616.

67 S. Scarmagnani, Z. Walsh, C. Slater, N. Alhashimy, B. Paull and D. Diamond, J. Mater. Chem., 2008, 2, 5063-5071.

68 S. Scarmagnani, Z. Walsh, F. B. Lopez, C. Slater, M. Macka, B. Paull and D. Diamond, e-J. Surf. Sci. Nanotechnol., 2009, 7, 649-652.

69 K. H. Fries, G. R. Sheppard, J. A. Bilbrey and J. Locklin, Polym. Chem., 2014, 5, 2094-2102.
70 S. Heng, X. Zhang, J. Pei and A. D. Abell, Biosensors, 2017, 7, 1-13.

71 S. Heng, C. A. Mcdevitt, R. Kostecki, J. R. Morey, B. A. Eijkelkamp, H. Ebendor, T. M. Monro and A. D. Abell, ACS Appl. Mater. Interfaces, 2016, 8, 6-11.

72 F. Benito-lopez, S. Scarmagnani, Z. Walsh, B. Paull, M. Macka and D. Diamond, Sensors Actuators B Chem., 2009, 140, 295-303.

73 R. J. Byrne, S. E. Stitzel and D. Diamond, J. Mater. Chem., 2006, 1332-1337.

74 L. Florea, A. Hennart, D. Diamond and F. Benito-lopez, Sens. Actuators, B, 2012, 175, 92-99.

75 L. Florea, D. Diamond and F. Benito-Lopez, Proc. 16th Int. Conf. Miniaturized Syst. Chem. Life Sci. MicroTAS, 2012, vol. 2012, pp. 1411-1413.

76 L. Florea, G. M. Guirk, F. Benito-Lopez and D. Diamond, 18th Int. Conf. Miniaturized Syst. Chem. Life Sci. MicroTAS, 2014, vol. 2014, pp. 1891-1893.

77 M. Natali, L. Soldi and S. Giordani, Tetrahedron, 2010, 66, $7612-7617$.

78 S. Scarmagnani, C. Slater, F. B. Lopez, D. Diamond, Z. Walsh, B. Paull and M. Macka, Int. J. Nanomanuf., 2010, 5, 38-52.

79 M. Baldrighi, G. Locatelli, J. Desper, C. B. Aakerçy and S. Giordani, Chem. - Eur. J., 2016, 22, 13976-13984.

80 K. H. Fries, J. D. Driskell, S. Samanta and J. Locklin, Anal. Chem., 2010, 82, 3306-3314.

81 H. Cui, H. Liu, S. Chen and R. Wang, Dyes Pigm., 2015, 115, 50-57.

82 L. Florea, A. Mckeon, D. Diamond and F. Benito-lopez, Langmuir, 2013, 29, 2790-2797. 